\title{
The Role of Calpains in Skeletal Muscle Remodeling with Exercise and Inactivity-induced Atrophy
}

\author{
Authors \\ Hayden W. Hyatt ${ }^{1} \mathbb{D}$, Scott K. Powers ${ }^{2}$
}

\section{Affiliations}

1 Applied Physiology and Kinesiology, University of Florida, Gainesville, United States

2 Applied Physiology, University of Florida, Gainesville, United States

\section{Key words}

calpain, disuse atrophy, proteolysis, muscle remodeling, membrane repair, exercise

accepted 24.05.2020

published online 17.07 .2020

\section{Bibliography}

Int J Sports Med 2020; 41: 994-1008

DOI 10.1055/a-1199-7662

ISSN $\quad 0172-4622$

(c) 2020. Thieme. All rights reserved.

Georg Thieme Verlag KG, Rüdigerstraße 14,

70469 Stuttgart, Germany

\section{Correspondence}

Dr. Hayden W. Hyatt

Applied Physiology and Kinesiology, University of Florida,

1864 Stadium Rd.,

32611-7011 Gainesville

United States

Tel.: +1 352294 1713, Fax : +1 3523925262

haydenhyatt@ufl.edu

\begin{abstract}
Calpains are cysteine proteases expressed in skeletal muscle fibers and other cells. Although calpain was first reported to act as a kinase activating factor in skeletal muscle, the consensus is now that calpains play a canonical role in protein turnover. However, recent evidence reveals new and exciting roles for calpains in skeletal muscle. This review will discuss the functions of calpains in skeletal muscle remodeling in response to both exercise and inactivity-induced muscle atrophy. Calpains participate in protein turnover and muscle remodeling by selectively cleaving target proteins and creating fragmented proteins that can be further degraded by other proteolytic systems. Nonetheless, an often overlooked function of calpains is that calpain-mediated cleavage of proteins can result in fragmented proteins that are biologically active and have the potential to actively influence cell signaling. In this manner, calpains function beyond their roles in protein turnover and influence downstream signaling effects. This review will highlight both the canonical and noncanonical roles that calpains play in skeletal muscle remodeling including sarcomere transformation, membrane repair, triad junction formation, regulation of excitation-contraction coupling, protein turnover, cell signaling, and mitochondrial function. We conclude with a discussion of key unanswered questions regarding the roles that calpains play in skeletal muscle.
\end{abstract}

\section{Introduction}

Skeletal muscle is a highly plastic tissue that undergoes continuous remodeling (i. e., plasticity), in response to changing levels of contractile activity (e. g., resistance exercise training-induced fiber hypertrophy or fiber atrophy resulting from prolonged periods of muscle inactivity). This skeletal muscle plasticity occurs due to the interaction between protein synthesis and protein degradation. In regard to the regulation of proteolysis, four major proteolytic systems exist in skeletal muscle (i. e. autophagy, ubiquitin-proteasome, caspase- 3 , and calpain systems) and accumulating evidence suggests that the calpain protease system plays a unique role in mediating skeletal muscle plasticity in response to both exercise and prolonged periods of muscle inactivity.

Calpains have been extensively studied since their discovery in skeletal muscle in 1964 [1]. The calpain family consists of a group of calcium $\left(\mathrm{Ca}^{2+}\right)$ activated proteases whose proteolytic function varies according to the calpain isoform and cell type. Calpains function through cleaving target substrates at specific sites, forming fragmented protein structures that can be further degraded by other proteolytic systems. However, truncation of calpain substrates by calpains can result in fragmented proteins with specific biological functions. Indeed, the first reported function of calpains in skeletal muscle was calpain-induced activation of phosphorylase kinase which resulted in this family of proteases being first termed as "kinase activating factors" [1,2]. Despite this early label as a signaling regulator, calpains are currently viewed by muscle physiologists as proteases that assist in myofibrillar protein turnover. Although calpains serve an important role in skeletal muscle protein turnover, accruing evidence reveals that calpains serve diverging roles in skeletal muscle fibers. 
This review will discuss the biological roles that calpains play in skeletal muscle. In particular, we will highlight how calpains facilitate the adaptive response of skeletal muscle fibers to chronic contractile activity (i. e. exercise) and muscle fiber atrophy associated with prolonged muscle inactivity. The first section of this review will introduce the calpain isoforms that are relevant to skeletal muscle and briefly discuss their general function and activation. We will then discuss the role that calpains play in skeletal muscle remodeling in response to both exercise training and in response to prolonged periods of skeletal muscle inactivity. Finally, we will also highlight the emerging evidence that calpains play an important signaling role in skeletal muscle.

\section{Calpains in Skeletal Muscle}

The calpain (CAPN) family of proteases in humans consists of 15 calpain isoforms, CAPN1-CAPN3 and CAPN5-CAPN16 with all isoforms containing a cysteine protease core [3]. Notably, CAPN4 is a $28 \mathrm{kd}$ subunit of calpain that lacks the cysteine proteolytic core. Due to this, CAPN4 is not recognized as an independent calpain isoform and has been reclassified as calpain subunit 1 (CAPNS1).

Three predominant calpain isoforms exist in skeletal muscle: 1 ) CAPN1; 2) CAPN2; and 3) CAPN3 [4]. Historically, CAPN1 and CAPN2 were previously referred to as $\mu$-calpain and m-calpain, respectively. The nomenclature of $\mu$-calpain and $\mathrm{m}$-calpain evolved from the levels of $\mathrm{Ca}^{2+}$ (the primary allosteric regulator of traditional calpains) required to activate calpains in vitro. Specifically, it was previously believed that activation of CAPN1 occurred when the cellular levels of free $\mathrm{Ca}^{2+}$ reached the $\mu \mathrm{M}$ range whereas CAPN2 was not activated until the intracellular $\mathrm{Ca}^{2+}$ concentrations reached the $\mathrm{mM}$ range. However, it has since been recognized that early calpain studies measuring the in vitro $\mathrm{Ca}^{2+}$ activation requirements did not reflect what occurred in vivo and thus, more appropriate nomenclature has been adopted $[5,6]$. Current understanding of the $\mathrm{Ca}^{2+}$ activation requirements for calpains will be discussed later in this review. Finally, CAPN3 is sometimes referred to as p94 in the literature. The use of p94 in reference to CAPN3 is due to its larger molecular weight of $94 \mathrm{kDa}$ compared to the $80 \mathrm{kDa}$ mass of CAPN1 and CAPN2.

\section{Overview of calpain structure and function}

CAPN1 and CAPN2 form heterodimers consisting of a similar, but distinct, $80 \mathrm{kDa}$ catalytic subunit (i.e. CAPN1 and CAPN2) and a $28 \mathrm{kDa}$ CAPNS1 regulatory subunit. In contrast, CAPN3 exists as a homodimer with two CAPN3 subunits. Note that while CAPN3 does have proteolytic function, several unique structural features of CAPN3 differentiate it from CAPN1 and CAPN2 (e. g. formation as a homodimer vs heterodimer) and allows CAPN3 to serve roles outside of proteolytic function; this topic will be discussed in more detail later in this review.

$\mathrm{Ca}^{2+}$ binding to calpain serves as an essential allosteric regulator for activating the proteolytic function of traditional calpains. The structural site on calpains responsible for proteolytic cleavage is labeled the catalytic triad; this catalytic triad is composed of an active site that is modulated by $\mathrm{Ca}^{2+}$. When $\mathrm{Ca}^{2+}$ is not bound to calpains the catalytic triad retains a structure that is not catalytically active [7]. Conversely, $\mathrm{Ca}^{2+}$ attachment to two $\mathrm{Ca}^{2+}$ binding sites on calpains results in a conformational change in the cysteine protease region of calpain and subsequent activation. A detailed discussion of the structure and mechanisms responsible for activation of calpains is beyond the goals of this review and readers are referred to other reports for more details [5, 7].

Importantly, the structural composition of calpains provide these proteases with the ability to selectively cleave specific sites on targeted proteins, as well as to cleave proteins that are unable to be accessed by other proteolytic systems (e. g. proteasome) [8]. In this manner, calpains play important roles for skeletal muscle protein turnover and can act independent and upstream of other proteolytic systems. Indeed, calpains were first noted as initiators of proteolytic degradation under post-mortem conditions whereby skeletal muscle ATP is depleted (thus preventing proteolysis occurring due to the ubiquitin-proteasome and autophagy systems which require ATP), yet protein degradation continued [8]. Curiously, it was observed that the protein degradation that occurred after the autophagy and ubiquitin proteasome systems were inactivated was due to $\mathrm{Ca}^{2+}$ leak from the sarcoplasmic reticulum (SR) and consequential activation of calpains $[8,9]$. This observation set the framework for our current understanding of calpains function in skeletal muscle.

Historically, calpains' primary role in skeletal muscle has been viewed as a facilitator of protein turnover through the proteolytic cleavage of sarcomeric and cytoskeletal proteins such as titin, dystrophin, nebulin, and desmin [7, 10,11]. However, our understanding of calpains has evolved to suggest that calpains participate in roles beyond protein turnover $[12,13]$. In this regard, the cleavage of select calpain substrates can result in biologically active fragments; these fragmented proteins can possess enzymatic activity or perform other biological functions. In this manner, calpains can function outside of their role in protein turnover and instead, influence cell signaling events leading to a diverse array of biological events (e.g., apoptosis). At present, direct evidence that calpains function outside the role of cytoskeletal proteolysis in skeletal muscle is limited. Despite this, abundant evidence exists in other cell types indicating that calpain acts in numerous roles including processes of cell motility, insulin signaling, and apoptosis. [14-18]. Further discussion of the expansive biological roles that calpain plays in skeletal muscle will be highlighted in future sections.

\section{Factors regulating proteolytic activity of calpains}

While $\mathrm{Ca}^{2+}$ is the primary regulator of the proteolytic activity of calpains, several other factors are also involved in regulating calpain activity. Key positive allosteric modifiers of calpain activity include autoproteolysis, binding of phospholipids, and phosphorylation. In contrast, the inhibitory protein calpastatin serves as the primary negative allosteric modifier of calpain activity. A brief overview of these allosteric regulators of calpain follow.

Autoproteolysis refers to the proteolytic removal of the N-terminal domain of calpains and is considered a key regulator of calpain activity [19]. In this regard, autoproteolysis allows for calpains to become activated at lower $\mathrm{Ca}^{2+}$ concentrations in the cell. For instance, autoprotoleysis of CAPN2 results in a 25 -fold reduction in the amount of $\mathrm{Ca}^{2+}$ required to elicit half-maximal activation [20]. Therefore, autoproteolysis is a key modulator of calpain activity. 
Autoproteolysis reduces the mass of the respective calpains, causing CAPN1 to appear as $76 \mathrm{kDa}$ and CAPN2 as $78 \mathrm{kDa}$ (compared to $80 \mathrm{kDa}$ for both CAPN1 and CAPN2 prior to autoproteolysis). Additionally, autoproteolysis of the $94 \mathrm{kDa}$ CAPN3 results in the autoproteolyzed fragment appearing at $\sim 55 \mathrm{kDa}$. Notably, the occurrence of autoproteoloysis is postulated to correspond with the initial activation of calpains. Due to this, the autolyzed forms of CAPN1 and CAPN2 are often used as markers of calpain activation in skeletal muscle [21].

Note that, although abundant evidence exists that autoproteolysis occurs within the calpain molecule, debate exists about the physiological importance of autoproteolysis in regulating calpain activity [5]. Autoproteolysis is posited to occur as an intermolecular reaction, thus requiring at least one calpain protein to become activated in order to cleave the N-terminal of nearby calpains [5]. However, many sites near the $\mathrm{N}$-terminal cleavage point are required for calpain function and would render calpains inactive if also cleaved. Evidence from in vitro studies suggest that these adjacent sites are also cleaved in a similar timed-fashion as the regulatory $\mathrm{N}$-terminal site during calpain activation [22]. In this regard, it has been argued that autoproteolysis can be an in vitro artifact in purified calpains due to their close proximity to one another [5]. Additionally, it has been argued that autoproteolysis would limit the ability to further regulate calpain activity due to the autoproteolyzed calpain being unable to return to a non-proteolyzed state. Nonetheless, autoproteolysis of calpain has been observed to occur in skeletal muscle and the relevance of autoproteolysis as a physiological regulator in vivo remains to be determined.

Calpain activity is also regulated by binding of phospholipids to calpains such as phosphatidylinositol [23]. For instance, exposure of calpains to phosphatidylinositol 4,5-bisphosphate $\left(\mathrm{PIP}_{2}\right)$ reduces the $\mathrm{Ca}^{2+}$ requirement for CAPN1 and CAPN2 autoproteolysis by three to five fold [24]. However, the concentrations of $\mathrm{PIP}_{2}$ required to induce this effect in vitro appear to be higher than what likely occurs in vivo [7]. Nonetheless, phospholipid binding in combination with other factors serves as additional mechanisms to regulate calpain activity.

Calpain activity is also regulated by its phosphorylation status. At least 6 phosphorylation sites have been identified on both CAPN1 and CAPN2 [7]. Phosphorylation of CAPN1 by protein kinase $A(P K A)$ can occur at several serine residues on CAPN1, resulting in increased calpain activity [25]. Conversely, dephosphorylation of CAPN1 by alkaline phosphatase decreases calpain activity [25]. However, PKA phosphorylation of CAPN2 results in decreased CAPN2 activity that is posited to occur due to the prevention of $\mathrm{PIP}_{2}$ binding [26]. To conclude, although phosphorylation of calpain can be a positive allosteric modifier of calpain activity, additional research is required to fully elucidate the complex role that phosphorylation plays in the regulation of calpain activity.

Finally, the key negative allosteric regulator of calpain activity is the endogenous protein, calpastatin. Calpastatin contains four inhibitory domains; only one domain is required to inhibit calpains. Thus, each calpastatin protein can inhibit up to four calpains [27]. More specifically, calpastatin inhibits calpain by binding to calpains and "looping" around the catalytic triad which blocks proteolytic cleavage from being able to occur [28]. Intriguingly, calpastatin is a calpain substrate itself and can be cleaved by calpain [29]. However, the looping maneuver that occurs during calpastatin-binding allows it to avoid the catalytic triad and prevents calpastatin from being cleaved in its calpain-bound state. The importance of calpastatin's regulation of calpains is likely demonstrated by the fact that the only known function of calpastatin is the inhibition of calpains [7]. The inhibitory actions of calpastatin on calpains are also regulated by several cellular events.

Interestingly, calpastatin-calpain binding only occurs when calpains become activated by $\mathrm{Ca}^{2+}$ binding. Considering that calpastatin only binds calpain in its activated state, it would appear likely that calpastatin functions as a mechanism to prevent prolonged calpain activation where more time has passed and allowed for calpastatin to associate and bind activated calpains. Calpastatin can also be phosphorylated by PKA and protein kinase C (PKC), although the effects of calpastatin phosphorylation are not well known [7].

\section{Modification of calpain substrates can increase their susceptibility for calpain-mediated degradation}

Robust evidence exists that post-transcriptional modifications of calpain substrates can modulate their susceptibility to degradation by calpains. For example, select kinases and phosphatases can regulate the susceptibility of specific calpain substrates to proteolytic degradation via phosphorylation or dephosphorylation [30]. For instance, PKC can phosphorylate troponin-I and increase its susceptibility for degradation by calpain [30]. The interconnection between calpains and phosphorylation as a regulating event is further illustrated by the fact that PKC is also a calpain substrate; however, calpain cleavage of PKC results in a catalytically active PKC fragment. Due to calpain cleavage increasing the kinase activity of PKC, the calpain-cleaved PKC fragments have an increased phosphorylation rate of calpain substrates and subsequently increases the susceptibility of calpain substrates to proteolytic degradation by calpains [31].

Further, oxidation of skeletal muscle proteins also increases their susceptibility to be cleaved by calpain due to conformational changes such as protein unfolding [32]. Oxidation of proteins affects their secondary and tertiary structure [33]. In this manner, protein unfolding increases the accessibility of calpains to access calpain cleavage target sites. Importantly, oxidative stress is a causative force in inactivity-induced muscle atrophy and the activation of calpains in inactivity-induced muscle atrophy will be discussed later in this review [34].

\section{Measurements of calpain activity}

Although several experimental approaches exist for measuring calpain activity, precise measurements of in vivo calpain activity remains challenging because of the allosteric regulation of calpains. For example, homogenizing skeletal muscle fibers releases calcium from the sarcoplasmic reticulum, resulting in calpain activation. Hence, this activation of calpain during the homogenization process masks the in vivo calpain activity that existed in the fibers prior to assay. Historically, calpain activity is measured in three ways: 1) zymography; 2) cleavage of exogenous fluorescent substrates; and 3) measurement of calpain-specific all-spectrin breakdown products. 
Zymography assays of calpain activity involve incubating homogenized tissue or cells with known calpain substrates and measuring the resulting cleavage products with gel electrophoresis. This technique can be useful for determining the factors that regulate calpain activity by adjusting variables within the assay (i. e. $\mathrm{Ca}^{2+}$ concentration) and observing the effects on calpain activity. However, these assays do not reflect in vivo calpain activity because of disruption of the cellular environment that calpains are exposed to during assay preparation. As mentioned previously, homogenizing muscle fibers disrupts the sarcoplasmic reticulum, releasing $\mathrm{Ca}^{2+}$ and activating calpains. Although this problem can be addressed by co-incubation with $\mathrm{Ca}^{2+}$ chelating agents (e. g., EGTA), it remains unclear how much of the released $\mathrm{Ca}^{2+}$ activates calpains during this process.

More recently, calpain activity has also been measured in situ by administering cell-permeable substrates that become fluorescent when cleaved by calpains. For instance, the synthetic construct 7-amino-4-chloromethylcoumarin-t-butoxycarbonyl-L-leucyl-Lmethionine amide (Boc-Leu-Met-CMAC) can cross skeletal muscle membranes and is subsequently transformed by glutathione S-transferase which makes it impermeable. This compound can then be cleaved by calpains resulting in a fluorescent chromophore that can be measured to reflect calpain activity [35]. However, this assay has several limitations that are independent of calpain activity such as the rate of thiol conjugation by glutathione S-transferase, the rate of substrate entry into the cell, and the intracellular concentration of the substrate.

Finally, specific biomarkers exist that can be measured as an index of calpain activity. Specifically, the universally expressed, membrane-associated cytoskeletal protein all-spectrin is cleaved by calpain resulting in a calpain-specific $150 \mathrm{kd}$ fragment that can be detected via western blot. The $150 \mathrm{kd}$ spectrin cleavage fragment has a relatively long half-life of $\sim 4.2 \mathrm{~h}$ and is widely-used for assessing the calpain activity that occurs in vivo[36, 37].

\section{Summary of structure and activation of calpains}

The unique proteolytic actions of calpains provides them the ability to perform several important roles in skeletal muscle tissue. Calpains are regulated by several allosteric regulators and cellular events that increase calpain's sensitivity to $\mathrm{Ca}^{2+}$ or increase the susceptibility of protein substrates to calpain-mediated degradation. While, historically, research on calpains' role in skeletal muscle has focused on degradation of sarcomeric proteins in muscle wasting conditions (e. g., inactivity-induced muscle atrophy), evidence also exists that calpains are activated following exercise. The next section focuses upon the role of calpain activation in skeletal muscle adaptations to exercise.

\section{Calpains and Exercise}

It is well-established that chronic exercise results in numerous adaptations to skeletal muscle fibers. In particular, skeletal muscle fibers undergo systematic remodeling in response to regular bouts of exercise; this occurs via a coordinated interaction between catabolic and anabolic reactions (i. e., increased protein degradation and increased protein synthesis) [38]. While four major proteolytic systems exist in skeletal muscle (i. e. the ubiquitin-proteasome, autophagy, caspase, and calpain systems), calpain is particularly interesting given that $\mathrm{Ca}^{2+}$, the primary allosteric regulator of calpain activity, is released from the SR in order to facilitate actin-myosin contractions during exercise. Thus, it is feasible that calpains become active during exercise training sessions that result in a prolonged increase in free $\mathrm{Ca}^{2+}$ in the cytosol; hence, if calpains are activated during exercise it is predicted that active calpain participates in skeletal muscle adaptation to exercise. The following sections highlight the evidence indicating that calpains are activated during exercise followed by a discussion of the physiological role that active calpains play in skeletal muscle remodeling in response to exercise training.

\section{Activation of calpains during exercise}

A long-debated question related to calpain activation during exercise is "do cytosolic levels of $\mathrm{Ca}^{2+}$ reach the level required to activate calpains?". In this regard, it is believed that the in vivo $\mathrm{Ca}^{2+}$ concentrations required to activate calpains occurs at $\sim 0.5-2 \mu \mathrm{M}$ for CAPN1 and $\sim 50-150 \mu \mathrm{M}$ for CAPN2 [7]. Resting free $\mathrm{Ca}^{2+}$ concentrations exist at $\sim 100 \mathrm{nM}$ in the cytosol of skeletal muscle and $\sim 390 \mu \mathrm{M}$ in the sarcoplasmic reticulum $[39,40]$. While measuring $\mathrm{Ca}^{2+}$ levels in contracting myofibers remains a difficult task, SR release of $\mathrm{Ca}^{2+}$ in response to an action potential in isolated muscle fibers has been measured at a peak increase of $\sim 120 \mu \mathrm{M}$ in slowtwitch muscle fibers and $\sim 358 \mu \mathrm{M}$ in fast-twitch muscle fibers [41]. Therefore, it is plausible that calpains are activated within these ranges of cytosolic $\mathrm{Ca}^{2+}$ concentrations. Further, it is possible that cytosolic $\mathrm{Ca}^{2+}$ concentrations in skeletal muscle may reach even higher levels during exercise because sustained $\mathrm{Ca}^{2+}$ levels within muscle fibers are determined by both the intensity and duration of exercise. Moreover, high intensity and/or eccentric exercise (i. e. muscle contractions during muscle lengthening) is capable of inducing damage to myofiber membranes allowing extracellular $\mathrm{Ca}^{2+}$ to enter the myofiber which often results in elevated levels of cytosolic $\mathrm{Ca}^{2+}$ for $12-36 \mathrm{~h}$ post exercise [42]. Therefore, when comparing experimental results regarding exercise-induced activation of calpain in skeletal muscles, it is important to consider the exercise protocol used in the experiments.

Calpain activation following exercise has predominantly been observed following two types of exercise modalities: prolonged endurance exercise and eccentric exercise. In this regard, several rodent experiments have demonstrated that calpain activity is increased following prolonged endurance exercise or continuous low frequency contractions [43-47]. In addition, evidence from rodent models also reveal that calpains are activated following eccentric exercise [48-51]. However, the evidence of calpain activation in humans following exercise is limited to a few studies that employ widely varying experimental protocols. A brief summary of these experiments follows.

Three independent human studies utilized autoproteolyzed calpain as a biomarker for calpain activation following exercise [5254]. In regard to the exercise modality, only one of these studies measured calpain activation following prolonged exercise; they reported no autoproteolysis of CAPN1 or CAPN3 immediately following a prolonged cycling exercise bout in a small number of trained cyclists [53]. A follow-up study demonstrated that eccentric exercise in humans resulted in autolyzed CAPN3 at $24 \mathrm{~h}$ following the 
completion of exercise with no evidence of calpain activation within the first three hours following exercise [52]. Importantly, these studies suffer from experimental shortcomings because both experiments used the cytosolic (i. e. soluble) fraction of muscle proteins to measure autoproteolysis of calpains. This is problematic because autoproteolyzed CAPN1 is concentrated within the myofibrillar fraction (i. e. insoluble) of rodent skeletal muscle proteins following both prolonged immobilization and endurance exercise $[45,55]$. Thus, use of the cytosolic muscle protein fraction to investigate calpain activation is likely a fatal experimental flaw. Indeed, a subsequent study illustrates this point with evidence that the observations of autolyzed CAPN3 in total human muscle homogenate is predominantly due to autoproteolysis of CAPN3 in the myofibrillar fraction following eccentric exercise [54].

Another study utilizing in vitro measures of calpain activity demonstrated that calpain activation occurred immediately following eccentric exercise in humans [56]. Calpain activity increased 3-fold immediately after a rigorous bout of eccentric exercise and increased calpain activity persisted for $95 \mathrm{~h}$ following completion of the exercise bout [56]. Notably, the majority of elevated calpain activity occurred in the myofibrillar (i. e. insoluble) fraction [56]. The fact that calpain activity is differentially regulated in various cell compartments within the myofiber highlights the need for stringent methodological approaches when measuring calpain activation in future studies.

Although few human studies have investigated the impact of prolonged endurance exercise on calpain activation, combining both the human and animal studies, it appears clear that calpains are activated in skeletal muscle in response to both prolonged endurance exercise and eccentric exercise [43-52, 54, 56, 57]. The following sections discuss the proteolytic roles that activated calpains serve in the skeletal muscle response to exercise, as well as a recently discovered nonproteolytic role of calpain with exercise.

\section{Proteolytic function of activated calpains with exercise}

Exercise-induced activation of calpains serve multiple roles in skeletal muscle. Calpains disassemble myofibrils in order to facilitate protein turnover of myofibrillar proteins and aid in remodeling with exercise training. Emerging evidence also suggests a role for calpains in the skeletal muscle response to exercise-induced damage. In this regard, calpains have recently been demonstrated to participate in membrane repair and altered excitation-contraction (EC) coupling. The following sections will discuss the proteolytic roles that calpains serve with exercise.

\section{Calpains and myofibrillar disassembly}

As introduced earlier, regular exercise training is well-known to induce skeletal muscle remodeling. For instance, chronic endurance exercise training results in a fast-to-slow shift in skeletal muscle fiber types. While the processes of muscle remodeling include a balance between protein synthesis and degradation, protein turnover is necessary to remove proteins in order for newly synthesized proteins to be incorporated. In this regard, myofibrils within skeletal muscle cells present a challenge for turnover of myofibrillar proteins due to its unique structure. The myofibril apparatus is tightly packed with myofilaments such as actin, myosin, tropomy- osin, troponin, and actinin. Myofibrils are dense in nature and this density limits diffusion of large proteins (i. e. > $200 \mathrm{kDa}$ ) within the myofibril. This is problematic for processes of protein turnover, as the proteasome complex, which is responsible for degradation of numerous muscle proteins, is unable to directly interact with intact myofibrils due to its large size of $\sim 2000 \mathrm{kDa}$. Instead, calpains facilitate myofibrillar protein turnover via cleavage of cytoskeletal proteins responsible for maintaining the structural integrity of myofibrils [58]. Calpain cleavage of these proteins functions to disassemble myofibrils, release myofibrillar proteins, and allow myofibrillar protein degradation by the ubiquitin-proteasome system ( Fig. 1a) [59]. Examples of the cytoskeletal proteins cleaved by calpains include $\alpha$-actinin, tropomyosin, desmin, nebulin, troponin, and titin.

In regard to the calpain-mediated cleavage of cytoskeletal proteins, one bout of exhaustive endurance excise in rodents increases calpain-mediated cleavage of tropomyosin and $\alpha$-actinin by $48 \%$ and $103 \%$, respectively [44]. $\alpha$-actinin anchors actin to the z-line and degradation of $\alpha$-actinin contributes to myofibrillar disassembly. The disassembly of the myofibril is believed to play an important role in removing damaged proteins and replacing them with newly synthesized proteins $[59,60]$. Moreover, disassembly likely plays an essential role for incorporating new myosin isoforms into the myofibril apparatus. Myosin heavy chains exist at $\sim 220 \mathrm{kDa}$ and fully assembled myosin molecules at $\sim 520 \mathrm{kDa}$ [59], and because of the large size of this molecule, myofibrillar disassembly is required to incorporate this large protein structure into the densely packed myofibrillar apparatus. In this manner, calpains may be necessary for muscle growth or fiber-type shifts in response to exercise training by facilitating myosin heavy chain incorporation into the myofibril apparatus. Notably, while substantial evidence exists that calpains play an important role in myofibril remodeling $[7,58,59,61]$, no studies have directly assessed the role of calpains in response to chronic exercise training. Therefore, while it appears likely that active calpains contribute to exercise-induced remodeling of skeletal muscles, a detailed understanding of the role that calpains play in training adaptations is not currently available. This topic remains an important area for future research.

\section{Calpains and exercise-induced damage}

Participation in eccentric or unaccustomed exercise often results in exercise-induced damage to skeletal muscle followed by a delayed onset of muscle soreness. Exercise-induced damage can be characterized by many factors such as sarcomere disruption (e.g. z-line streaming), damaged cytoskeletal proteins (e.g. titin, desmin, dystrophin, etc.), diminished membrane integrity, compromised EC-coupling, and decreased force output [62-64]. Recovery from exercise-induced damage typically occurs over the span of a few days and results in skeletal muscle adaptations that help prevent future damage against a similar exercise bout: known as the repeated bout effect [65]. Recovery from exercise-induced damage requires protein turnover of dysfunctional proteins and their replacement by newly synthesized proteins, hence calpains would likely play an important role. The impact of exercise on protein turnover following damaging exercise is demonstrated by the fact that whole body protein breakdown can remain elevated for 10 days following a single bout of eccentric exercise [66]. 


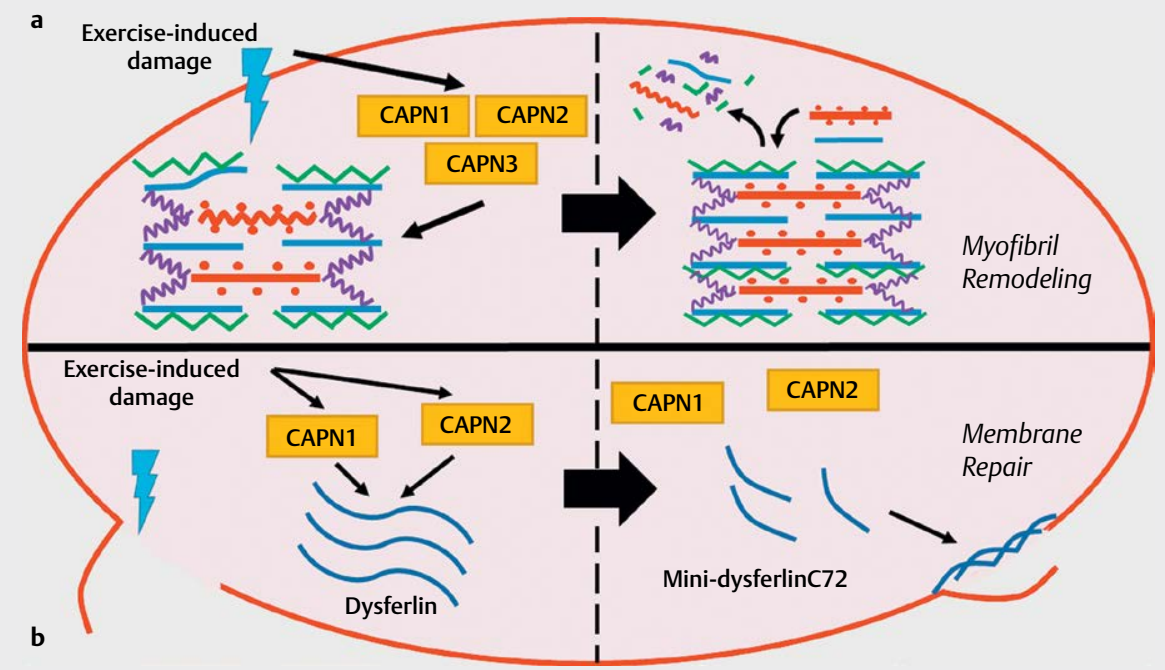

- Fig. 1 a Exercise-induced damage activates calpains and results in degradation of cytsoskeletal proteins encapsulating contractile proteins in order for contractile apparatus dissassembly and repair. $\mathbf{b}$ Exercise-induced damage causes membrane damage. Resulting activation of calpains causes cleavage of dysferlin into mini-dysferlin to create lattice for membrane repair. See text for more details.

In regard to the mechanisms responsible for exercise-induced damage, mechanical sheer forces resulting in microscopic tears and subsequent oxidative damage are plausible events that have been proposed to explain the occurrence of exercise-induced damage (e. g. disrupted z-line streaming) [67]. In addition to these postulates, recent evidence also implicates calpain activation as a direct contributor to exercised-induced muscle injury. For example, a study utilizing ex vivo eccentric muscle contractions demonstrated that pharmacological inhibition of calpains diminishes biomarkers of eccentric exercise-induced muscle damage [51]. Specifically, inhibition of calpain activity prevented eccentric exercise-induced disruption of muscle sarcolemma integrity and loss of cytoskeletal proteins. Moreover, inhibition of calpain activation blunted the eccentric exercise-induced reductions in muscle force generating capacity. The authors posit that eccentric exercise-induced activation of calpains results in the cleavage of cytoskeletal proteins and the disruption of myofiber membrane integrity which are characteristic of exercise-induced damage. A separate group utilized in situ eccentric contractions and showed that pharmacological blockade of calpain activation resulted in faster recovery of force production at 3 days following eccentric contractions [49]. Together, these studies suggest that activation of calpains are causal in exercise-induced muscle damage.

Given that calpain inhibition can protect against reductions in muscle force production following exercise-induced muscle injury, it appears that calpain activation is a contributory event to muscle damage. Nonetheless, an alternative interpretation is that calpain activation occurs in response to exercise-induced "micro-damage" to skeletal muscle and that calpains degrade damaged proteins and facilitate the recovery from injury, instead of causing the actual damage per se. Indeed, calpains have been postulated to play important roles in responding to micro-damage in skeletal muscle fibers and facilitating sarcolemma repair $[68,69]$. A brief discussion of the role that calpains play in sarcolemma repair follows.

\section{Calpains facilitate membrane repair}

Emerging evidence indicates that calpains can contribute to muscle fiber membrane damage resulting from damaging exercise ( $\vee$ Fig. 1b). In this regard, both CAPN1 and CAPN2 play an essential role in mediating membrane repair following muscle damage [68]. Specifically, CAPN1 and CAPN2 contribute to the repair process of skeletal muscle membranes by cleaving the protein dysferlin into mini-dysferlin $\mathrm{C} 72_{2}$ [69]. Mini-dysferlin ${ }_{\mathrm{C} 72}$, but not full length dysferlin, acts to localize to the damaged membrane site and recruit mitsugumin 53 (MG53), forming a lattice that stabilizes the injured site and further recruits repair machinery. The injured membrane site results in $\mathrm{Ca}^{2+}$-induced activation of CAPN1 and CAPN2 leading to the required cleavage of dysferlin into mini-dysferlin ${ }_{C 72}$. Importantly, this evidence highlights the important concept that calpains role is not limited to protein turnover; indeed, active calpains are capable of cleaving proteins and altering their biological function to facilitate processes such as membrane repair.

Calpains participation in membrane repair via cleavage of dysferlin likely has important indications for exercise-induced membrane damage. This is due to findings that dysferlin-knockout mice show a significantly blunted repair rate with eccentric exercise-induced membrane damage [70]. A lack of dysferlin following eccentric exercise would prevent calpains from cleaving dysferlin and creating mini-dysferlin ${ }_{\mathrm{C} 72}$ which is important in the membrane repair process. Thus, it is predicted that calpain cleavage of dysferlin into mini-dysferlin ${ }_{\mathrm{C} 72}$ is a key event in the cellular response to damaging exercise. Collectively, these findings support the idea that calpains respond to micro-damage following exercise and facilitate muscle repair instead of causing detrimental cell damage. Given this, it may also be plausible that calpains protect against further 
muscle damage by reducing the force generating capacity of skeletal muscle via alterations in EC-coupling.

\section{Calpains affect EC-coupling by degrading triad junction proteins}

As mentioned previously, calpains are purported to play a role in the decreased force output that occurs following exercise-induced muscle damage. While it is established that diminished EC-coupling contributes to exercise damage-induced reductions in muscle force production, the mechanisms responsible for this phenomenon remain poorly understood. Previous studies suggest that eccentric exercise impairs EC-coupling potentially through decreases in the abundance of triad proteins $[71,72]$. In this regard, recent evidence has implicated that calpains play a significant role in diminished ECcoupling following exercise-induced damage through remodeling of the triad junction. Triad junctions are sites where $T$ tubules and the SR connect to facilitate the release of $\mathrm{Ca}^{2+}$ in response to an action potential. Thus, the triad junction is an essential element to EC-coupling [73].

Pharmacological inhibition of calpains prevents eccentric exercise-induced decreases in the protein abundance of the triad junction proteins dihydropyridine receptor (DHPR), junctophilin 1 (JP1), and junctophilin 2 (JP2) following in situ eccentric exercise in rats [49]. Importantly, preventing the degradation of triad junction proteins by inhibiting calpains also coincided with conserved force production three days after the eccentric exercise bout. Junctophilins span across the t-tubule membrane and tether the SR to form the triad junction and are essential for triad junction formation. Indeed, junctophilin-knockout mice display greatly reduced triad junction formation and die soon after birth [74]. Thus, it appears likely that calpain-mediated reductions in the tethering components of the triad junction (i. e. junctophillins) and voltage sensing proteins responsible for facilitating $\mathrm{Ca}^{2+}$ release from the SR (i.e. DHPR) would result in the diminished EC-coupling that occurs with exercise-induced damage ( $\triangleright$ Fig. 2 a).

The cleavage of triad proteins by calpains as a means to facilitate further proteolytic degradation of these proteins follows the canonical view of calpains' primary function. In addition, calpain initiation of decreased EC-coupling would also prevent the force that muscle fibers would experience during recovery (i. e. due to lower contractile capacity) and would reduce the potential for further damage occurring. Thus, calpain cleavage of triad junction proteins may serve a protective function that prevents exercisedamaged muscles from producing high levels of contractile forces until muscle recovery has occurred. While proteolytic remodeling of the triad junction by calpains serves an important role following exercise-induced damage, recent discoveries have indicated that calpains also play a unique role within the triad junction that is completely independent of their proteolytic function.

\section{Non-proteolytic function of calpain in exercise}

Recent evidence reveals that CAPN3 is an essential structural component for conformation of the triad junction ( Fig. 1b) [75]. Moreover, the structural involvement of CAPN3 at the triad junction plays an important role in the adaptive response of skeletal muscle to endurance exercise [76]. Notably, the concept that CAPN3 can function in a role other than proteolysis was first noted in patients with limb girdle muscle dystrophy type 2A (LGMD2A). LGMD2A develops due to mutations in the CAPN3 gene. Intriguingly, assessment of the localization of mutations within the CAPN3 gene revealed that several patients with LGMD2A had mutations in regions of the gene that did not affect the proteolytic activity of CAPN3 [77]. This discovery led to the development of knock-in mice containing a mutant CAPN3 gene that renders CAPN3 proteolytically inactive but structurally intact [78]. It was observed that CAPN3 KO mice lacked appropriate triad junction formation, how-

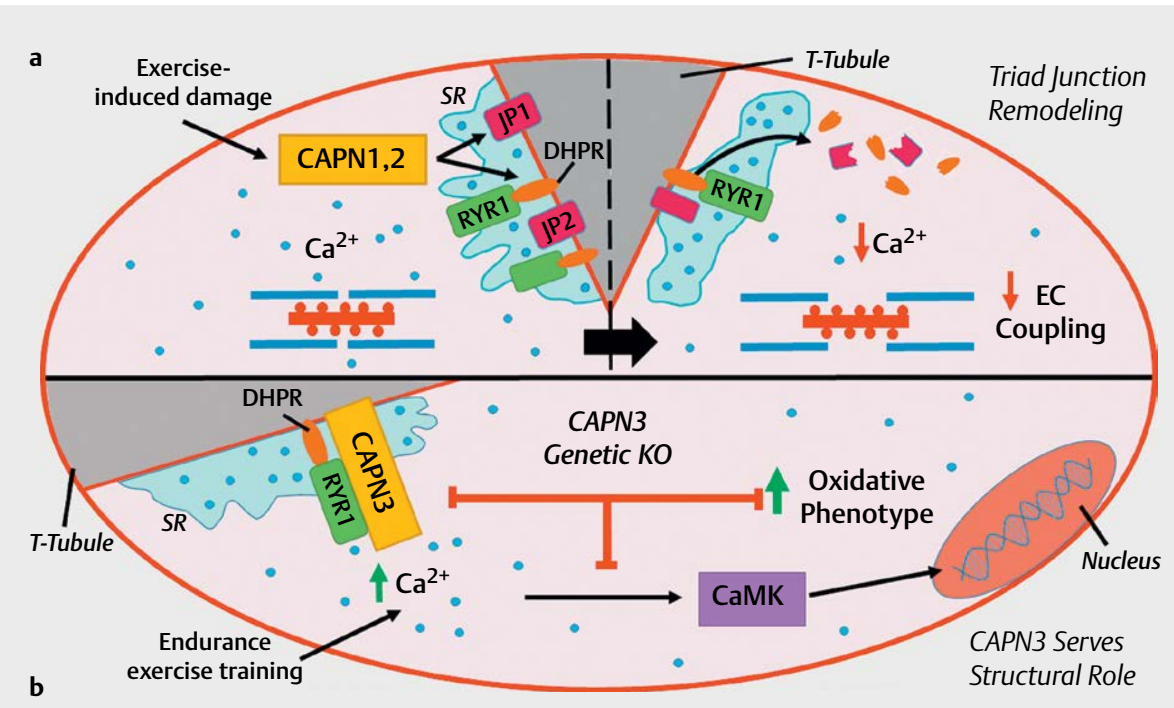

-Fig. 2 a Exercise-induced damage activates calpains and cleaves triad junction proteins resulting in decreased excitation-contraction (EC) coupling. b CAPN3 plays a structural role in triad junction formation in order to facilitate $\mathrm{Ca}^{2+}$ release required for $\mathrm{CaMK}$ activation and downstream adaptations. See text for more details. (Abbreviations: CAPN-Calpain, RyR- ryanodine receptor, JP1/2- junctophilin proteins, DHPR- dihydropyrodine receptor, CaMK- calmodulin dependent kinase). 
ever mutant mice with proteolytically inactive CAPN3 formed triad junctions with functioning $\mathrm{Ca}^{2+}$ release channels. Thus, it was apparent that the proteolytic activity of CAPN3 was not responsible for triad junction formation, but that CAPN3 was acting in a separate role. This finding led to the discovery that CAPN3 functions as a structural component that tethers the triad junction to connectin/titin within skeletal muscle [78].

In regard to exercise, the structural role of CAPN3 appears essential in the adaptive response to endurance exercise [76]. For instance, CAPN3 KO mice have reduced presence of ryanodine receptor (RyR) and, consequentially, impaired $\mathrm{Ca}^{2+}$ release from the SR [75]. This is problematic, as $\mathrm{Ca}^{2+}$ is a key signal for adaptations with exercise training. While endurance exercise training normally promotes transcription of genes involved in oxidative metabolism and a slow-twitch muscle fiber phenotype [79], CAPN3 KO mice have an impaired response to endurance training and exhibit blunted expression of genes associated with the slow-fiber phenotype [76]. Due to the structural role of CAPN3 in formation of the triad junction, its absence reduces RyR presence and blunts $\mathrm{Ca}^{2+}$ signaling. In turn, CAPN3 KO mice lack appropriate signaling through the $\mathrm{Ca}^{2+}$-calmodulin-dependent protein kinase (CaMK) pathway which is responsible for upregulated transcription of genes involved in oxidative metabolism [76]. Therefore, CAPN3 appears to be essential in the promotion of a slow-twitch phenotype in skeletal muscle in response to endurance exercise training. The novel finding that CAPN3 serves as a structural component required for exerciseinduced adaptations highlights the important role that calpains play outside of their canonical function in proteolysis.

\section{Summary of calpains in exercise and future directions}

While research on the role that calpains play in exercise-induced adaptations remains in its infancy, growing evidence suggests that calpains have an important role in exercise-induced adaptation in skeletal muscle. Indeed, calpains have a large impact in the skeletal muscle response to eccentric exercise. Nonetheless, future studies are required to determine if calpain activation is a detrimental consequence of eccentric exercise, or if calpains play an essential role to the muscle adaptations that coincide with exercise training.

From a resistance training perspective, compared to concentric exercise, eccentric exercise is postulated to elicit a greater hypertrophy response purportedly due to the greater load placed on skeletal muscle fibers [80]. Given the role of calpains in skeletal muscle remodeling, it is plausible that calpain activation by eccentric exercise may also play a critical role in these higher "returns" for a given exercise bout. In addition, accruing evidence suggests that the role of calpains in muscle adaptation extends beyond its involvement in proteolysis. Indeed, calpains contribution to membrane repair and triad junction formation highlight that calpains are not limited to their function in proteolysis and may have untold roles in skeletal muscle function that have yet to be discovered. Future research is needed in order to fully elucidate the role of calpains in skeletal muscle and its adaptation to exercise. In the next section, we discuss the role that calpains play in promoting skeletal muscle atrophy during prolonged periods of muscle inactivity.

\section{Calpains in Inactivity-induced Muscle Atrophy}

Skeletal muscle atrophy is a conserved biological process that is essential for systemic proteostasis and energy balance in times of need (e. g. prolonged fasting). Skeletal muscle comprises nearly $40 \%$ of human body weight, $\sim 75 \%$ of total body protein content, and skeletal muscle maintenance alone can contribute to $20 \%$ of resting energy expenditure [81]. Skeletal muscle mass is regulated in response to the activity load it experiences in order to meet the "needs" of the body but not exceed those means in order to maintain efficient homeostasis. In this regard, skeletal muscle responds to periods of muscle inactivity by initiating processes that facilitate muscle atrophy. Inactivity-induced muscle atrophy occurs as a result of conditions that significantly decrease the work performed by skeletal muscle such as limb-immobilization, prolonged bedrest, and mechanical ventilation-induced inactivity of inspiratory muscles [12, 82-87]. Unfortunately, prolonged inactivity results in skeletal muscle atrophy that is detrimental due to a reduced forcegenerating capacity of skeletal muscle. Severe muscle atrophy can be a devastating consequence that can impair the livelihood of those affected by reducing the ability to perform tasks of daily living and in some patient populations, muscle atrophy can increase the risk of morbidity and mortality [88]. In the subsequent sections, we will discuss both the tools utilized to study inactivity-induced muscle atrophy and the role that calpains play in mediating atrophy during prolonged muscle inactivity.

\section{Experimental models and measures of inactivity- induced muscle atrophy}

Because of the clinical significance of skeletal muscle atrophy, scientific interest in investigating the mechanism has increased markedly; however, investigating the mechanisms responsible for inactivity-induced muscle atrophy in humans remains a challenge for several reasons. For example, human studies seeking to delineate the mechanisms of inactivity-induced atrophy require muscle biopsies that are invasive and difficult to obtain in some forms of muscle inactivity-induced muscle atrophy (e. g. biopsies from diaphragm muscle in patients undergoing mechanical ventilation). Further, the amount of muscle tissue obtained in most muscle biopsies is small (e. g., 50-100 mg) and limits the number of analyses that can be performed on as single sample. Additionally, human experimental models to study inactivity-induced muscle atrophy such as prolonged bed-rest and limb-casting are expensive to perform. Moreover, many of the experimental interventions utilized to delineate the roles of signaling pathways (e. g. pharmacological inhibitors, transgenic gene modulation, etc.) are not approved for use in humans. Therefore, because of these complications in the study of inactivity-induced atrophy in humans, animal models to study inactivity-induced muscle atrophy have been developed. For instance, hindlimb-unloading via suspension of a rodent's tail can be utilized to unweight the hindlimbs and simulate the muscle atrophy that occurs during bed-rest or space flight. Limb casting in rodents can be utilized to immobilize limbs and promote the skeletal muscle atrophy that occurs due to casting in humans. Denervation of skeletal muscle motor neurons in animals is also utilized to emulate muscle inactivity that occurs with spinal cord injury. Ad- 
ditionally, rodent models of mechanical ventilation exist that decrease the contractile activity of inspiratory muscles (i. e. diaphragm) and result in inactivity-induced muscle atrophy similar to what occurs in humans undergoing mechanical ventilation. All of these animal models have been successfully used to investigate the mechanisms responsible for inactivity-induced muscle atrophy.

Inactivity-induced muscle atrophy is measured by reductions in muscle mass or the cross-sectional area of muscle and individual muscle fibers. Other hallmarks of inactivity-induced muscle atrophy include alterations in the ultrastructure of muscle (e. g. disrupted z-line streaming, altered mitochondrial morphology, etc.) and reductions in the specific force production of muscle (i. e., force produced per cross-sectional area of muscle) [12, 89, 90]. Importantly, calpains have been implicated to play a mechanistic role in inactivity-induced muscle atrophy.

\section{Evidence of calpain involvement in inactivity- induced muscle atrophy}

Several lines of evidence indicate that calpains play an important role in the occurrence of inactivity-induced muscle atrophy. For instance, pharmacological inhibition of calpains attenuates muscle atrophy that occurs in rodents during both limb casting and prolonged mechanical ventilation [12,90]. However, it may be important to note that while calpain inhibitors are capable of inhibiting a broad array of calpain isoforms, current and previous generations of calpain inhibitors often exhibit off-target effects such as inhibition of cathepsins (i. e. lysosomal proteolytic enzymes). Although current generations of calpain inhibitors have markedly improved selectivity of calpains over cathepsins [91], the potential for offtarget effects of most calpain inhibitors remains a concern in interpretation of data from studies using these inhibitors. Instead, transgenic overexpression of calpastatin, the endogenous inhibitor of calpains, is a highly selective approach for inhibiting calpains in skeletal muscle. Indeed, the only known function of calpastatin is the inhibition of calpains which makes calpastatin overexpression a powerful experimental tool to selectively target calpain activation. In this regard, two separate studies have demonstrated that transgenic mouse models overexpressing calpastatin are resistant to inactivity-induced muscle atrophy provoked by hindlimb unloading [89, 92].

Collectively, strong evidence exists that calpains play a key role in the muscle atrophy that occurs in response to prolonged muscle inactivity. The following paragraphs will further elaborate the currently available evidence on how calpains become activated during muscle inactivity and the specific functions that activated calpains may serve in inactivity-induced muscle atrophy.

\section{Regulators of increased calpain activity during inactivity-induced muscle atrophy}

Prolonged inactivity in skeletal muscle results in several events that lead to calpain activation. For example, prolonged muscle inactivity (i. e., hindlimb unloading and denervation) result in significant increases of cytosolic levels of $\mathrm{Ca}^{2+}$ leading to calpain activation $[93,94]$. Additionally, muscle inactivity results in increased production of reactive oxygen species (ROS) and the oxidation of calpain substrates which increases their susceptibility to degradation [32]. Moreover, phosphorylation of calpain target substrates also con- tributes to increased degradation by calpains [94]. The following paragraphs will discuss these points in more detail.

Inactivity induces calpain activation due to increased cytosolic free $\mathrm{Ca}^{2+}$

Cytosolic $\mathrm{Ca}^{2+}$ levels increase during prolonged muscle inactivity and result in the activation of calpains $[93,94]$. For instance, one study utilizing denervation as a model of muscle inactivity showed that cytosolic $\mathrm{Ca}^{2+}$ levels were increased 7 days following denervation and this rise in $\mathrm{Ca}^{2+}$ levels coincided with the time-point of calpain activation [94]. The mechanism(s) to explain why prolonged muscle inactivity results in increases in cytosolic levels of calcium has been a challenging issue to investigate. However, emerging evidence points to two potential mechanisms.

One mechanism of increased muscle $\mathrm{Ca}^{2+}$ levels with inactivity is through an inward flux of extracellular $\mathrm{Ca}^{2+}$ due to opening of $\mathrm{Ca}^{2+}$ ion channels. Transient receptor potential, subfamily $\mathrm{V}$, member 2 (TRPV2) and purinergic ionotropic $P 2 X_{7}$ receptor $\left(P 2 X_{7} R\right)$ channels are upregulated with denervation in rodents and become activated during prolonged muscle inactivity [95]. Therefore, activation of extracellular $\mathrm{Ca}^{2+}$ channels would contribute to increased free $\mathrm{Ca}^{2+}$ levels with inactivity and lead to activation of calpains.

Another possibility that could explain elevated $\mathrm{Ca}^{2+}$ levels during prolonged muscle inactivity is the occurrence of $\mathrm{Ca}^{2+}$ leak from the SR. For example, evidence reveals that the SR in diaphragm muscle becomes "leaky" during prolonged mechanical ventilation resulting in unregulated $\mathrm{Ca}^{2+}$ release [96]. The occurrence of $\mathrm{Ca}^{2+}$ leak from the SR is linked with oxidative stress that occurs with prolonged muscle inactivity. Prolonged inactivity in skeletal muscles is associated with increased ROS production leading to oxidative stress $[34,97,98]$. In this manner, increased ROS production results in oxidation of the RYR 1 and dissociation of calstabin 1 from the RYR1 complex [96]. This is important because Calstabin 1 stabilizes the RYR1 complex and its dissociation results in $\mathrm{Ca}^{2+}$ leak during muscle inactivity; however, antioxidant treatment prevents the disassociation of calstabin 1 and mitigates SR $\mathrm{Ca}^{2+}$ leak with inactivity [96]. Therefore, oxidization of the RYR1 complex and resultant $\mathrm{Ca}^{2+}$ leak from the SR during muscle inactivity would serve as one mechanism to activate calpains and induce their proteolytic functions. Notably, ROS also oxidize calpain target substrates and increase their susceptibility for cleavage by calpains. Indeed, posttranscriptional modifications such as oxidation and phosphorylation of calpain substrates can increase the susceptibility of degradation by calpains $[32,94]$.

\section{Oxidation and phosphorylation of target substrates increases calpain degradation}

ROS-mediated oxidation of calpain substrate proteins results in a higher susceptibility to calpain cleavage [32]. Oxidation of proteins results in alterations in the secondary or tertiary structures of proteins such as protein unfolding; the unfolding of oxidized calpain substrates increases the accessibility of the calpain proteolytic core to cleavage sites within substrate proteins. As mentioned, skeletal muscle inactivity results in increased oxidant production $[34,97,98]$. While many sources of ROS exist during muscle inactivity, mitochondria are noted to become damaged in skeletal mus- 
cle during prolonged inactivity and are a key source of elevated ROS emissions [99]. Increased emission of ROS from mitochondria results in protein oxidation and higher cleavage rates of these oxidized proteins by calpains. Further, increased production of ROS in skeletal muscles also increases calpain activation. Specifically, provision of a mitochondrial targeted antioxidant prevents an increase in calpain activity in skeletal muscles during both mechanical ventilation and limb-immobilization [97, 98]. Importantly, preventing increased mitochondrial ROS emissions and subsequent calpain activation also protects against muscle atrophy due to prolonged inactivity $[97,98]$. Hence, increased ROS emissions in skeletal muscles during long periods of inactivity plays an important role in calpain activation.

Phosphorylation of calpain substrates during muscle inactivity is another factor that increases calpain degradation of proteins. For instance, recent evidence reveals that phosphorylation of desmin by glycogen synthase kinase $3-\beta$ (CSK3- $\beta$ ) substantially increases its degradation by CAPN1 in skeletal muscle in response to denervation [94]. Conversely, inhibition of GSK3- $\beta$ by expression of a dominant negative GSK3- $\beta$ prevented the degradation of desmin. Thus, phosphorylation of calpain substrates also likely play a key role in determining a proteins' susceptibility to calpain cleavage.

\section{Summary of calpain activation with muscle inactivity}

Collectively, increased calpain activity during muscle inactivity can occur by increases in intracellular $\mathrm{Ca}^{2+}$ levels. Increased intracellular $\mathrm{Ca}^{2+}$ levels serve to activate calpains and can happen due to $\mathrm{Ca}^{2+}$ leak from the SR and/or influx of extracellular $\mathrm{Ca}^{2+}$ owing to activation of membrane $\mathrm{Ca}^{2+}$ ion channels. Additionally, allosteric modification of calpain substrates via oxidation or phosphorylation can also increase the susceptibility of calpain substrates to cleavage and result in an increased proteolytic cleavage of target substrates.

\section{Calpains role in inactivity-induced muscle atrophy}

Skeletal muscle atrophy occurs when muscle protein degradation rates exceed the rate of protein synthesis. Protein degradation is facilitated by four major proteolytic systems in skeletal muscle: 1 ) autophagy; 2) ubiquitin-proteasome; 3) caspase-3; and 4) calpain systems. While all four proteolytic systems are activated in skeletal muscle during prolonged periods of inactivity [99], each proteolytic system likely plays a different role in promoting muscle atrophy. Both the caspase- 3 and calpain systems function to cleave their targeted proteins to form fragmented sections while the autophagy and ubiquitin-proteasome systems further breakdown proteins and protein fragments to amino acids. While calpains are unable to fully degrade their target proteins, the cleavage of these proteins has marked effects on skeletal muscle function and proteostasis.

Inactivity-induced activation of calpains disrupt skeletal muscle ultrastructure

As mentioned earlier in this review, disruptions in the ultrastructure of skeletal muscle, such as disrupted z-line streaming, are often observed with inactivity-induced muscle atrophy [100,101]. The streaming of z-lines is an important factor for effectively transmitting force throughout the length of the muscle fiber. Intriguingly, exposure of skeletal muscle to high levels of $\mathrm{Ca}^{2+}$ results in near disappearance of z-lines [102]. Indeed, many of calpains' substrates (e.g. desmin, nebulin, titin, etc.) are responsible for structural integrity and force transmission of muscle fibers. For instance, desmin is responsible for attaching the z-disk to the sarcolemma, nebulin anchors actin to the z-line, and titin acts as a molecular spring that facilitates passive elasticity. Thus, calpain cleavage of its substrates disrupt muscle ultrastructure during inactivity-induced muscle atrophy. In turn, calpain-induced alterations in muscle ultrastructure would also contribute to decrements in force production observed with inactivity. In this regard, calpastatin overexpressing mice that underwent 14 days of hindlimb unloading had complete protection against reductions in soleus muscle specific force [89]. Importantly, this protection in muscle specific force coincided with preserved sarcomere structure (i. e. z-line streaming) [89]. Furthermore, pharmacological inhibition of calpains prevents the decrease in muscle-specific force that occur due to mechanical ventilation [90]. These studies suggest that calpains facilitate the disruptions in muscle fiber ultrastructure and diminished force production, which have long been regarded as hallmarks in inactivityinduced muscle atrophy. In addition, calpain-mediated turnover of myofibrillar proteins may also contribute to reductions in the force generating capacity of inactive muscles.

\section{Calpains initiate protein degradation that contributes to muscle atrophy}

As mentioned earlier in this review, calpains play important roles in myofibril disassembly. Myofibril disassembly is necessary in order for myofibrillar proteins to be degraded during the atrophic process. Recall that the proteasome complex is unable to degrade proteins within intact myofibrils due to spatial limitations imposed by the large size of the proteasome complex and the densely packed myofibrillar apparatus [59]. In contrast, calpains' size and selective cleavage of specific calpain substrates provide them the ability to cleave proteins responsible for maintaining the dense structure of the myofibril apparatus.

Activated calpains cleave sarcomeric proteins (e. g. desmin, nebulin, titin) that encapsulate the myofibril and result in freeing of myofibrillar proteins $[10,11,103]$. Indeed, actin and myosin are released from the myofibrillar apparatus in a $\mathrm{Ca}^{2+}$ dependent manner and this observation was first noted over four decades ago [104]. Today, calpain-mediated disassembly of myofibrils is considered the rate limiting factor for ubiquitin-proteasome degradation of contractile proteins [105]. The important role that calpains serve in initiating protein degradation is demonstrated by pharmacological and transgenic studies in rodents showing that inhibition of calpains attenuates inactivity-provoked muscle atrophy due to limb immobilization, hindlimb unloading, and mechanical ventilation $[12,90,92]$.

In addition to myofibril disassembly, recent evidence has also indicated that calpains can also cleave contractile proteins. While calpains do not readily degrade undenatured forms of actin and myosin $[7,11]$, recent evidence indicates that oxidation of actin and myosin increases their susceptibility to degradation by calpains [32]. Given that prolonged-inactivity induces oxidative stress in skeletal muscle, calpains may also function to participate in break- 
down of actin and myosin during inactivity-induced muscle atrophy.

Collectively, the activation of calpains leads to the cleavage of sarcomeric and cytoskeletal proteins that results in the disassembly of myofibrils. Myofibril disassembly functions to allow degradation of myofibrillar proteins via the proteasome complex. Furthermore, during periods of oxidative stress in skeletal muscle (e. g., prolonged muscle inactivity), calpains also assist in degradation of contractile proteins such as actin and myosin. Therefore, calpains contribute to muscle atrophy during inactivity by accelerating muscle proteolysis ( $\mathbf{F i g}$. 3 ).

\section{Activated calpains promote atrophic signaling}

The specificity of calpains' cleavage mechanism provides this unique protease with the ability to cleave proteins to form biologically active protein fragments with altered function. In this manner, calpains have the capacity to serve as upstream signaling regulators. Accruing evidence has revealed that calpains are signaling effectors for numerous cellular processes in several types of nonmuscle cells [14-17]. However, our understanding of calpain cleavage resulting in downstream signaling events in skeletal muscle is not well understood. Nonetheless, specific calpain cleavage products may act as mechanisms for mediating muscle atrophy [90]. Examples of calpain signaling in skeletal muscle fibers follows.

An important pathway of calpain signaling is the calpain-mediated cleavage of pro-caspase- 3 into its active form, caspase-3. Following cleavage, activated caspase- 3 initiates numerous downstream signaling events [106]. For example, activated caspase-3 degrades proteins involved in DNA repair such as poly(ADP-ribose) polymerase (PARP), as well as contractile proteins such as actin and myosin $[107,108]$. The effects of calpain cleaved caspase-3 on DNA repair and proteolysis highlights an important capability of active calpain.

Calpains also participate in cell signaling events by influencing mitochondrial function. For example, calpain-mediated cleavage of the BCL-2 family member, Bid, results in its truncated form known as tBid. Evidence in cardiac myocytes reveals that tBid can translocate to the mitochondria and recruit Bax; this leads to permeabilization of the mitochondrial membrane and increased ROS production $[109,110]$. As mentioned previously, increased ROS production plays an important role in the occurrence of inactivityinduced atrophy and dysfunctional mitochondria are the major contributor [99]. Similar to cardiac myocytes, calpain cleavage of Bid into tBid occurs in diaphragm muscle during prolonged mechanical ventilation and the appearance of tBid is prevented with calpain inhibition [90]. Therefore, it is plausible that calpain formation of tBid may play an important role in the causation of the mitochondrial dysfunction that occurs with muscle inactivity.

Calpain also participates in cell signaling by cleaving apoptosisinducing factor (AIF) in mitochondria [111]. AIF is an important signaling molecule that is capable of inducing cell death. The proteolytic activity of calpains is required for AIF to be released from mitochondria where it then translocates to the nucleus and causes chromatin condensation and DNA degradation. Although AIF plays important roles in signaling for cell death pathways, intact AIF also serves an important role in mitochondrial function. Indeed, muscle-specific loss of AIF results in mitochondrial dysfunction and muscle fiber atrophy [112]. Although it is currently unclear if calpain cleavage of AIF contributes to inactivity-induced muscle atrophy, cytosolic levels of AIF are increased with denervation and hindlimb unloading $[107,113]$. Therefore, it is feasible that calpain cleavage of AIF and subsequent release from mitochondria may play a role in inactivity-induced muscle atrophy.

The relationship between calpains and mitochondrial function is further demonstrated by the existence of a mitochondrial targeting motif on CAPN1 [114]. CAPN1 has been shown to translocate and accumulate in mitochondria of cardiac myoctes resulting in mitochondrial dysfunction [115]. However, it currently remains unknown if CAPN1 enters the mitochondria in skeletal muscle. If CAPN1 is capable of translocating to mitochondria within skeletal muscle, it is plausible that calpain-mediated cleavage of Bid, AIF, and CAPN1 targeting of mitochondria may contribute to the mito-

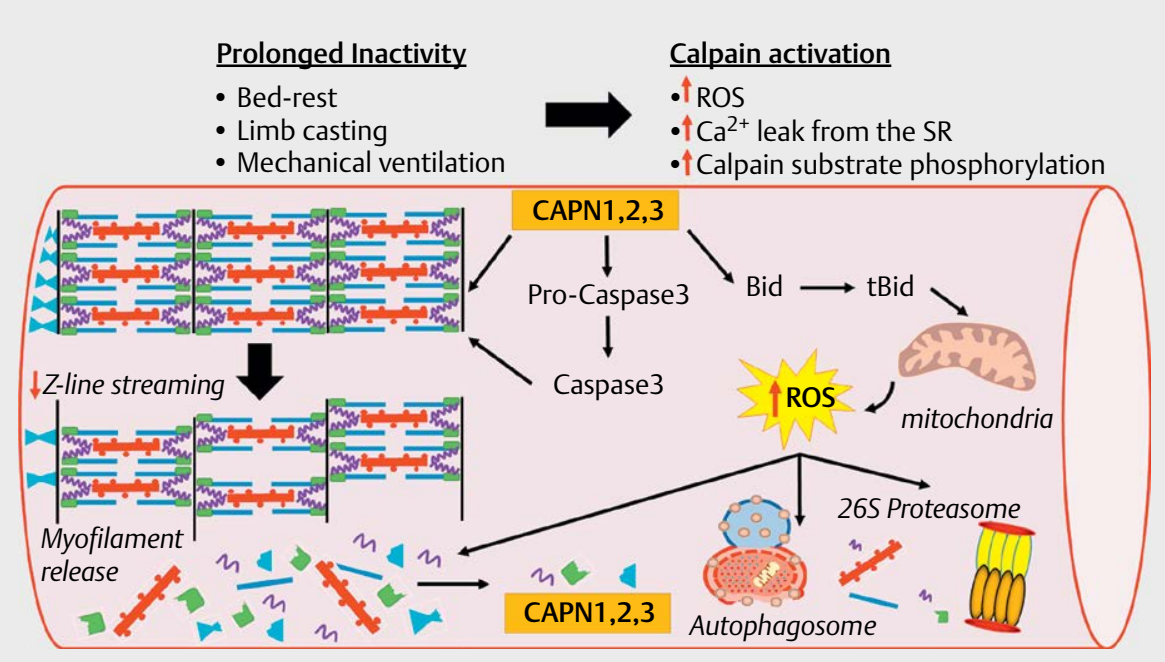

Fig. 3 Prolonged inactivity induces muscle atrophy via activation of calpains. Activated calpains cause dissassembly of the contractile apparatus, disruptions in z-line streaming, activation of caspase system, mitochondrial dysfunction, and increased proteolysis. See text for more details. 
chondrial dysfunction that occurs in skeletal muscle during prolonged inactivity.

A final example of the importance of calpain signaling is the discovery that calpain activation results in decreased Akt activity in skeletal muscle [116]. Although the mechanism(s) responsible for this observation remain unclear, Akt abundance is not decreased in skeletal muscle indicating that calpain-mediated downregulation of Akt activity is likely due to decreased phosphorylation [117]. Regardless of the mechanisms responsible for calpain-mediated downregulation of Akt, the finding that active calpains decrease Akt signaling is another important example of how active calpains plays a key signaling role in skeletal muscles and highlights the importance of future research on this topic.

\section{Conclusions and Future Directions}

Accumulating evidence indicates that calpains play important roles in skeletal muscle remodeling in response to both exercise training and inactivity-induced muscle atrophy. While calpains have been traditionally viewed as a simple proteolytic step in the processes of protein turnover, exciting new evidence reveals that calpains serve roles in skeletal muscle fibers that extend beyond their canonical involvement in cleavage of proteins. For instance, calpains contribute to the cellular adaptation to stressors by cleaving proteins and altering their biological function (e. g., calpain cleavage of dysferlin contributing to membrane repair). Further, calpains also function in roles independent of their proteolytic functions such as the structural involvement of calpains in the conformation of triad junctions. Therefore, the accumulating evidence that calpains have biological functions beyond protein turnover highlights the importance of broadening the scope of studies into calpain function.

While the study of skeletal muscle calpains may offer untapped potential, it is important to note that several limitations remain in the study of calpain biology. In an effort to stimulate future work in this field, the remainder of this section will focus on these limitations and how addressing them will lead to heightened understanding of calpain function. First, measuring calpain activity in vivo remains a difficult task due to the multitude of factors involved in regulating calpains; improvements in this area will allow investigators to further establish the physiological paradigms that result in activation of calpains (e. g. prolonged exercise in humans). In addition, the tools utilized to identify calpain-truncated proteins is currently complex and time-consuming. While computational models that predict calpain-cleavage sites are improving, our current understanding of the specific structural components that dictate calpain cleavage sites within calpain substrates is still limited. Further development of predictive computational algorithms would allow for researchers to more easily identify calpain-truncated proteins. Indeed, the current methodology utilized for identification of calpain-truncated proteins has occurred largely through recognition of these fragmented proteins via western blotting followed by biochemical identification of the cleaved fragment. Due to the laborious efforts currently required to identify calpain-mediated fragments, future development of predictive modeling in tandem with tools such as mass spectrometry would lead to the development of databases capable of identifying biologically im- portant targets at a larger and more efficient scale. This would then require in vivo studies to assess the physiological impact of specific calpain-truncated proteins. In this regard, the significance of calpain-truncated proteins has already begun to emerge in studies of non-muscle cells. For example, it was recently discovered that calpain-mediated tau fragmentation is associated with disease progression in Alzheimer's [118]. Hence, the study of calpains and calpain-truncated proteins may yield new-insight in skeletal muscle biology. While current dogma suggests that calpains are simple "cleavers" in the proteolytic chain, this perception evolved from their first proclaimed functions as "kinase activating factors" [2]. Perhaps it is time to once again revisit the role of calpains in skeletal muscle physiology.

\section{Funding}

This work was supported by a grant from the National Institutes of Health (NIH R21 AR073956) awarded to SKP.

\section{Conflict of Interest Statement}

The current review meets the ethical standards of the International Journal of Sports Medicine as discussed in Harriss et al. [119]. The authors declare that they no conflicts of interest.

\section{References}

[1] Meyer WL, Fischer EH, Krebs EG. Activation of skeletal muscle phosphorylase B kinase by Ca. Biochemistry 1964; 3: 1033-1039

[2] Huston RB, Krebs EG. Activation of skeletal muscle phosphorylase kinase by $\mathrm{Ca} 2+$. II. Identification of the kinase activating factor as a proteolytic enzyme. Biochemistry 1968; 7: 2116-2122

[3] Ono Y, Saido TC, Sorimachi H. Calpain research for drug discovery: Challenges and potential. Nat Rev Drug Discov 2016; 15: 854-876. doi:10.1038/nrd.2016.212

[4] Murphy RM. Calpains, skeletal muscle function and exercise. Clin Exp Pharmacol Physiol 2010; 37: 385-391. doi:10.1111/j.14401681.2009.05310.x

[5] Campbell RL, Davies PL. Structure-function relationships in calpains. Biochem J 2012; 447: 335-351. doi:10.1042/BJ20120921

[6] Edmunds T, Nagainis PA, Sathe SK et al. Comparison of the autolyzed and unautolyzed forms of mu- and m-calpain from bovine skeletal muscle. Biochim Biophys Acta 1991; 1077: 197-208

[7] Goll DE, Thompson VF, Li H et al. The calpain system. Physiol Rev 2003; 83: 731-801. doi:10.1152/physrev.00029.2002

[8] Koohmaraie M. The role of $\mathrm{Ca}(2+)$-dependent proteases (calpains) in post mortem proteolysis and meat tenderness. Biochimie 1992; 74 : 239-245

[9] Bartoli M, Richard I. Calpains in muscle wasting. Int J Biochem Cell Biol 2005; 37: 2115-2133. doi:10.1016/j.biocel.2004.12.012

[10] Purintrapiban J, Wang MC, Forsberg NE. Degradation of sarcomeric and cytoskeletal proteins in cultured skeletal muscle cells. Comp Biochem Physiol B Biochem Mol Biol 2003; 136: 393-401

[11] Huang J, Forsberg NE. Role of calpain in skeletal-muscle protein degradation. Proc Natl Acad Sci USA 1998; 95: 12100-12105. doi:10.1073/pnas.95.21.12100 
[12] Talbert EE, Smuder A], Min K et al. Calpain and caspase-3 play required roles in immobilization-induced limb muscle atrophy. J Appl Physiol (1985) 2013; 114: 1482-1489. doi:10.1152/japplphysiol.00925.2012

[13] Spencer MJ, Guyon JR, Sorimachi $\mathrm{H}$ et al. Stable expression of calpain 3 from a muscle transgene in vivo: immature muscle in transgenic mice suggests a role for calpain 3 in muscle maturation. Proc Natl Acad Sci USA 2002; 99: 8874-8879. doi:10.1073/pnas.132269299

[14] Cortesio CL, Boateng LR, Piazza TM et al. Calpain-mediated proteolysis of paxillin negatively regulates focal adhesion dynamics and cell migration. J Biol Chem 2011; 286: 9998-10006. doi:10.1074/jbc.M110.187294

[15] Tan Y, Wu C, De Veyra T et al. Ubiquitous calpains promote both apoptosis and survival signals in response to different cell death stimuli. J Biol Chem 2006; 281: 17689-17698. doi:10.1074/jbc. M601978200

[16] Yuasa T, Amo-Shiinoki K, Ishikura $S$ et al. Sequential cleavage of insulin receptor by calpain 2 and gamma-secretase impairs insulin signalling. Diabetologia 2016; 59: 2711-2721. doi:10.1007| s00125-016-4102-5

[17] Beltran L, Chaussade C, Vanhaesebroeck B et al. Calpain interacts with class IA phosphoinositide 3-kinases regulating their stability and signaling activity. Proc Natl Acad Sci USA 2011; 108: 16217-16222. doi:10.1073/pnas. 1107692108

[18] Carragher NO. Calpain inhibition: A therapeutic strategy targeting multiple disease states. Curr Pharm Des 2006; 12: 615-638. doi: $10.2174 / 138161206775474314$

[19] Baki A, Tompa P, Alexa A et al. Autolysis parallels activation of mu-calpain. Biochem J 1996; 318 (Pt 3): 897-901. doi:10.1042/ bj3180897

[20] Nagainis PA, Wolfe FH, Sathe SK et al. Autolysis of the millimolar $\mathrm{Ca} 2+$-requiring form of the $\mathrm{Ca} 2+$-dependent proteinase from chicken skeletal muscle. Biochem Cell Biol 1988; 66: 1023-1031

[21] Murphy RM, Verburg E, Lamb GD. Ca2 + activation of diffusible and bound pools of mu-calpain in rat skeletal muscle. J Physiol 2006; 576: 595-612. doi:10.1113/jphysiol.2006.114090

[22] Chou JS, Impens F, Gevaert $\mathrm{K}$ et al. $\mathrm{m}$-Calpain activation in vitro does not require autolysis or subunit dissociation. Biochim Biophys Acta 2011; 1814: 864-872. doi:10.1016/j.bbapap.2011.04.007

[23] Coolican SA, Hathaway DR. Effect of L-alpha-phosphatidylinositol on a vascular smooth muscle $\mathrm{Ca} 2+$-dependent protease. Reduction of the Ca2 + requirement for autolysis. J Biol Chem 1984; 259 : $11627-11630$

[24] Cong J, Goll DE, Peterson AM et al. The role of autolysis in activity of the $\mathrm{Ca} 2+$-dependent proteinases (mu-calpain and m-calpain). J Biol Chem 1989; 264: 10096-10103

[25] Du M, Li X, Li Z et al. Phosphorylation regulated by protein kinase A and alkaline phosphatase play positive roles in mu-calpain activity. Food Chem 2018; 252: 33-39. doi:10.1016/j.foodchem.2018.01.103

[26] Smith SD, Jia Z, Huynh KK et al. Glutamate substitutions at a PKA consensus site are consistent with inactivation of calpain by phosphorylation. FEBS Lett 2003; 542: 115-118. doi:10.1016/ s0014-5793(03)00361-2

[27] Hanna RA, Garcia-Diaz BE, Davies PL. Calpastatin simultaneously binds four calpains with different kinetic constants. FEBS Lett 2007; 581: 2894-2898. doi:10.1016/j.febslet.2007.05.035

[28] Hanna RA, Campbell RL, Davies PL. Calcium-bound structure of calpain and its mechanism of inhibition by calpastatin. Nature 2008; 456: 409-412. doi:10.1038/nature07451

[29] Porn-Ares MI, Samali A, Orrenius S. Cleavage of the calpain inhibitor, calpastatin, during apoptosis. Cell Death Differ 1998; 5: 1028-1033. doi:10.1038/sj.cdd.4400424
[30] Di Lisa F, De Tullio R, Salamino F et al. Specific degradation of troponin $\mathrm{T}$ and $\mathrm{I}$ by mu-calpain and its modulation by substrate phosphorylation. Biochem J 1995; 308 ( Pt 1): 57-61. doi:10.1042/ bj3080057

[31] Kishimoto A, Mikawa K, Hashimoto K et al. Limited proteolysis of protein kinase $C$ subspecies by calcium-dependent neutral protease (calpain). J Biol Chem 1989; 264: 4088-4092

[32] Smuder AJ, Kavazis AN, Hudson MB et al. Oxidation enhances myofibrillar protein degradation via calpain and caspase-3. Free Radic Biol Med 2010; 49: 1152-1160. doi:10.1016/j.freeradbiomed.2010.06.025

[33] Davies KJ, Delsignore ME. Protein damage and degradation by oxygen radicals. III. Modification of secondary and tertiary structure. J Biol Chem 1987; 262: 9908-9913

[34] Powers SK, Smuder A], Judge AR. Oxidative stress and disuse muscle atrophy: cause or consequence? Curr Opin Clin Nutr Metab Care 2012; 15: 240-245. doi:10.1097/MCO.0b013e328352b4c2

[35] Gailly P, De Backer F, Van Schoor M et al. In situ measurements of calpain activity in isolated muscle fibres from normal and dystrophinlacking mdx mice. J Physiol 2007; 582: 1261-1275. doi:10.1113/ jphysiol.2007.132191

[36] Wang KK, Posmantur R, Nath R et al. Simultaneous degradation of alphall- and betall-spectrin by caspase 3 (CPP32) in apoptotic cells. J Biol Chem 1998; 273: 22490-22497. doi:10.1074/jbc.273.35.22490

[37] Yan XX, Jeromin A, Jeromin A. Spectrin breakdown products (SBDPs) as potential biomarkers for neurodegenerative diseases. Curr Transl Geriatr Exp Gerontol Rep 2012; 1: 85-93. doi:10.1007/s13670-0120009-2

[38] Russell AP. Molecular regulation of skeletal muscle mass. Clin Exp Pharmacol Physiol 2010; 37: 378-384. doi:10.1111/j.14401681.2009.05265.x

[39] Williams DA, Head SI, Bakker A] et al. Resting calcium concentrations in isolated skeletal muscle fibres of dystrophic mice. J Physiol 1990; 428: 243-256. doi:10.1113/jphysiol.1990.sp018210

[40] Ziman AP, Ward CW, Rodney GG et al. Quantitative measurement of $\mathrm{Ca}(2)(+)$ in the sarcoplasmic reticulum lumen of mammalian skeletal muscle. Biophys J 2010; 99: 2705-2714. doi:10.1016/j. bpj.2010.08.032

[41] Baylor SM, Hollingworth S. Sarcoplasmic reticulum calcium release compared in slow-twitch and fast-twitch fibres of mouse muscle. J Physiol 2003; 551: 125-138. doi:10.1113/jphysiol.2003.041608

[42] Armstrong RB, Warren GL, Warren JA. Mechanisms of exerciseinduced muscle fibre injury. Sports Med 1991; 12: 184-207. doi:10.2165/00007256-199112030-00004

[43] Raj DA, Booker TS, Belcastro AN. Striated muscle calcium-stimulated cysteine protease (calpain-like) activity promotes myeloperoxidase activity with exercise. Pflugers Arch 1998; 435: 804-809. doi: $10.1007 / \mathrm{s} 004240050587$

[44] Belcastro AN. Skeletal muscle calcium-activated neutral protease (calpain) with exercise. J Appl Physiol (1985) 1993; 74: 1381-1386. doi:10.1152/jappl.1993.74.3.1381

[45] Arthur GD, Booker TS, Belcastro AN. Exercise promotes a subcellular redistribution of calcium-stimulated protease activity in striated muscle. Can J Physiol Pharmacol 1999; 77: 42-47. doi:10.1139/ cjpp-77-1-42

[46] Sultan KR, Dittrich BT, Leisner E et al. Fiber type-specific expression of major proteolytic systems in fast- to slow-transforming rabbit muscle. Am J Physiol Cell Physiol 2001; 280: C239-C247. doi:10.1152/ajpcell.2001.280.2.C239

[47] Gissel H. Ca2 + accumulation and cell damage in skeletal muscle during low frequency stimulation. Eur J Appl Physiol 2000; 83: 175-180. doi:10.1007/s004210000276 
[48] Kanzaki K, Kuratani M, Matsunaga S et al. Three calpain isoforms are autolyzed in rat fast-twitch muscle after eccentric contractions. J Muscle Res Cell Motil 2014; 35: 179-189. doi:10.1007/s10974-0149378-9

[49] Kanzaki K, Watanabe D, Kuratani M et al. Role of calpain in eccentric contraction-induced proteolysis of $\mathrm{Ca}(2+)$-regulatory proteins and force depression in rat fast-twitch skeletal muscle. J Appl Physiol (1985) 2017; 122: 396-405. doi:10.1152/japplphysiol.00270.2016

[50] Zhang BT, Whitehead NP, Gervasio OL et al. Pathways of $\mathrm{Ca}(2)(+)$ entry and cytoskeletal damage following eccentric contractions in mouse skeletal muscle. J Appl Physiol (1985) 2012; 112: 2077-2086. doi:10.1152/japplphysiol.00770.2011

[51] Zhang BT, Yeung SS, Allen DG et al. Role of the calcium-calpain pathway in cytoskeletal damage after eccentric contractions. J Appl Physiol (1985) 2008; 105: 352-357. doi:10.1152/japplphysiol.90320.2008

[52] Murphy RM, Goodman CA, McKenna MJ et al. Calpain-3 is autolyzed and hence activated in human skeletal muscle $24 \mathrm{~h}$ following a single bout of eccentric exercise. J Appl Physiol (1985) 2007; 103: 926-931. doi:10.1152/japplphysiol.01422.2006

[53] Murphy RM, Snow R], Lamb GD. mu-Calpain and calpain-3 are not autolyzed with exhaustive exercise in humans. Am J Physiol Cell Physiol 2006; 290: C116-C122. doi:10.1152/ajpcell.00291.2005

[54] Murphy RM, Vissing K, Latchman $\mathrm{H}$ et al. Activation of skeletal muscle calpain- 3 by eccentric exercise in humans does not result in its translocation to the nucleus or cytosol. J Appl Physiol (1985) 2011; 111: 1448-1458. doi:10.1152/japplphysiol.00441.2011

[55] Vermaelen M, Sirvent P, Raynaud F et al. Differential localization of autolyzed calpains 1 and 2 in slow and fast skeletal muscles in the early phase of atrophy. Am J Physiol Cell Physiol 2007; 292: C1723-C731. doi:10.1152/ajpcell.00398.2006

[56] Raastad T, Owe SG, Paulsen G et al. Changes in calpain activity, muscle structure, and function after eccentric exercise. Med Sci Sports Exerc 2010; 42: 86-95. doi:10.1249/MSS.0b013e3181ac7afa

[57] Bartoli M, Bourg N, Stockholm D et al. A mouse model for monitoring calpain activity under physiological and pathological conditions. J Biol Chem 2006; 281: 39672-39680. doi:10.1074/jbc.M608803200

[58] Neti G, Novak SM, Thompson VF et al. Properties of easily releasable myofilaments: are they the first step in myofibrillar protein turnover? Am J Physiol Cell Physiol 2009; 296: C1383-C1390. doi:10.1152/ ajpcell.00022.2009

[59] Goll DE, Neti G, Mares SW et al. Myofibrillar protein turnover: the proteasome and the calpains. J Anim Sci 2008; 86(14 Suppl): E19-E35. doi:10.2527/jas.2007-0395

[60] Kumar V, Atherton P, Smith K et al. Human muscle protein synthesis and breakdown during and after exercise. J Appl Physiol (1985) 2009; 106: 2026-2039. doi:10.1152/japplphysiol.91481.2008

[61] Lametsch R, Roepstorff P, Moller HS et al. Identification of myofibrillar substrates for mu-calpain. Meat Sci 2004; 68: 515-521. doi:10.1016/j.meatsci.2004.03.018

[62] Morgan DL, Allen DG. Early events in stretch-induced muscle damage. J Appl Physiol (1985) 1999; 87: 2007-2015. doi:10.1152/ jappl.1999.87.6.2007

[63] Proske U, Morgan DL. Muscle damage from eccentric exercise: mechanism, mechanical signs, adaptation and clinical applications. J Physiol 2001; 537: 333-345. doi:10.1111/j.1469-7793.2001.00333.x

[64] Takekura H, Fujinami N, Nishizawa T et al. Eccentric exercise-induced morphological changes in the membrane systems involved in excitation-contraction coupling in rat skeletal muscle. J Physiol 2001; 533: 571-583. doi:10.1111/j.1469-7793.2001.0571a.x

[65] McHugh MP. Recent advances in the understanding of the repeated bout effect: the protective effect against muscle damage from a single bout of eccentric exercise. Scand J Med Sci Sports 2003; 13: 88-97
[66] Fielding RA, Meredith CN, O'Reilly KP et al. Enhanced protein breakdown after eccentric exercise in young and older men. J Appl Physiol (1985) 1991; 71: 674-679. doi:10.1152/jappl.1991.71.2.674

[67] Owens DJ, Twist C, Cobley JN et al. Exercise-induced muscle damage: What is it, what causes it and what are the nutritional solutions? Eur J Sport Sci 2019; 19: 71-85. doi:10.1080/17461391.2018.1505957

[68] Mellgren RL, Miyake K, Kramerova I et al. Calcium-dependent plasma membrane repair requires $\mathrm{m}$ - or mu-calpain, but not calpain-3, the proteasome, or caspases. Biochim Biophys Acta 2009; 1793: 1886-1893. doi:10.1016/j.bbamcr.2009.09.013

[69] Lek A, Evesson FJ, Lemckert FA et al. Calpains, cleaved mini-dysferlinC72, and L-type channels underpin calcium-dependent muscle membrane repair. J Neurosci 2013; 33: 5085-5094. doi:10.1523/ JNEUROSCI.3560-12.2013

[70] Roche JA, Lovering RM, Bloch RJ. Impaired recovery of dysferlin-null skeletal muscle after contraction-induced injury in vivo. Neuroreport 2008; 19: 1579-1584. doi:10.1097/WNR.0b013e328311ca35

[71] Ingalls CP, Warren GL, Zhang JZ et al. Dihydropyridine and ryanodine receptor binding after eccentric contractions in mouse skeletal muscle. J Appl Physiol (1985) 2004; 96: 1619-1625. doi:10.1152/ japplphysiol.00084.2003

[72] Corona BT, Balog EM, Doyle JA et al. Junctophilin damage contributes to early strength deficits and EC coupling failure after eccentric contractions. Am J Physiol Cell Physiol 2010; 298: C365-C376. doi:10.1152/ajpcell.00365.2009

[73] Franzini-Armstrong C, Jorgensen AO. Structure and development of E-C coupling units in skeletal muscle. Ann Rev Physiol 1994; 56 : 509-534. doi:10.1146/annurev.ph.56.030194.002453

[74] Ito K, Komazaki S, Sasamoto K et al. Deficiency of triad junction and contraction in mutant skeletal muscle lacking junctophilin type 1. J Cell Biol 2001; 154: 1059-1067. doi:10.1083/jcb.200105040

[75] Kramerova I, Kudryashova E, Wu B et al. Novel role of calpain-3 in the triad-associated protein complex regulating calcium release in skeletal muscle. Hum Mol Genet 2008; 17: 3271-3280. doi:10.1093/ hmg/ddn223

[76] Kramerova I, Kudryashova E, Ermolova $\mathrm{N}$ et al. Impaired calcium calmodulin kinase signaling and muscle adaptation response in the absence of calpain 3. Hum Mol Genet 2012; 21: 3193-3204. doi: $10.1093 / \mathrm{hmg} / \mathrm{dds} 144$

[77] Milic A, Daniele N, Lochmuller H et al. A third of LGMD2A biopsies have normal calpain 3 proteolytic activity as determined by an in vitro assay. Neuromuscul Disord 2007; 17: 148-156. doi:10.1016/j. nmd.2006.11.001

[78] Ojima K, Ono Y, Ottenheijm C et al. Non-proteolytic functions of calpain-3 in sarcoplasmic reticulum in skeletal muscles. J Mol Biol 2011; 407: 439-449. doi:10.1016/j.jmb.2011.01.057

[79] Demirel HA, Powers SK, Naito $\mathrm{H}$ et al. Exercise-induced alterations in skeletal muscle myosin heavy chain phenotype: dose-response relationship. J Appl Physiol (1985) 1999; 86: 1002-1008. doi:10.1152/jappl.1999.86.3.1002

[80] Hody S, Croisier JL, Bury T et al. Eccentric Muscle Contractions: Risks and Benefits. Front Physiol 2019; 10: 536. doi:10.3389/ fphys.2019.00536

[81] Rasmussen BB, Phillips SM. Contractile and nutritional regulation of human muscle growth. Exerc Sport Sci Rev 2003; 31: 127-131

[82] Powers SK, Duarte JA, Le Nguyen B et al. Endurance exercise protects skeletal muscle against both doxorubicin-induced and inactivityinduced muscle wasting. Pflugers Arch 2019; 471: 441-453. doi:10.1007/s00424-018-2227-8

[83] Shanely RA, Zergeroglu MA, Lennon SL et al. Mechanical ventilationinduced diaphragmatic atrophy is associated with oxidative injury and increased proteolytic activity. Am J Respir Crit Care Med 2002; 166: 1369-1374. doi:10.1164/rccm.200202-0880C 
[84] LeBlanc AD, Schneider VS, Evans H] et al. Regional changes in muscle mass following 17 weeks of bed rest. J Appl Physiol (1985) 1992; 73: 2172-2178. doi:10.1152/jappl.1992.73.5.2172

[85] Jones SW, Hill RJ, Krasney PA et al. Disuse atrophy and exercise rehabilitation in humans profoundly affects the expression of genes associated with the regulation of skeletal muscle mass. FASEB J 2004; 18: 1025-1027. doi:10.1096/fj.03-1228fje

[86] Ferrando AA, Lane HW, Stuart CA et al. Prolonged bed rest decreases skeletal muscle and whole body protein synthesis. Am J Physiol 1996; 270: E627-E633. doi:10.1152/ajpendo.1996.270.4.E627

[87] Levine S, Nguyen T, Taylor $\mathrm{N}$ et al. Rapid disuse atrophy of diaphragm fibers in mechanically ventilated humans. N Engl J Med 2008; 358: 1327-1335. doi:10.1056/NEJMoa070447

[88] Rantanen T. Muscle strength, disability and mortality. Scand J Med Sci Sports 2003; 13: 3-8

[89] Salazar J], Michele DE, Brooks SV. Inhibition of calpain prevents muscle weakness and disruption of sarcomere structure during hindlimb suspension. J Appl Physiol (1985) 2010; 108: 120-127. doi:10.1152/japplphysiol.01080.2009

[90] Nelson WB, Smuder AJ, Hudson MB et al. Cross-talk between the calpain and caspase- 3 proteolytic systems in the diaphragm during prolonged mechanical ventilation. Crit Care Med 2012; 40: 1857-1863. doi:10.1097/CCM.0b013e318246bb5d

[91] Siklos M, BenAissa M, Thatcher GR. Cysteine proteases as therapeutic targets: does selectivity matter? A systematic review of calpain and cathepsin inhibitors. Acta Pharm Sin B 2015; 5: 506-519. doi:10.1016/j.apsb.2015.08.001

[92] Tidball JG, Spencer M]. Expression of a calpastatin transgene slows muscle wasting and obviates changes in myosin isoform expression during murine muscle disuse. J Physiol 2002; 545: 819-828. doi:10.1113/jphysiol.2002.024935

[93] Ingalls CP, Wenke JC, Armstrong RB. Time course changes in [Ca2+]i, force, and protein content in hindlimb-suspended mouse soleus muscles. Aviat Space Environ Med 2001; 72: 471-476

[94] Aweida D, Rudesky I, Volodin A et al. GSK3-beta promotes calpain1-mediated desmin filament depolymerization and myofibril loss in atrophy. J Cell Biol 2018; 217: 3698-3714. doi:10.1083/ jcb.201802018

[95] Cea LA, Cisterna BA, Puebla C et al. De novo expression of connexin hemichannels in denervated fast skeletal muscles leads to atrophy. Proc Natl Acad Sci USA 2013; 110: 16229-16234. doi:10.1073/ pnas. 1312331110

[96] Matecki S, Dridi H, Jung B et al. Leaky ryanodine receptors contribute to diaphragmatic weakness during mechanical ventilation. Proc Natl Acad Sci USA 2016; 113: 9069-9074. doi:10.1073/pnas.1609707113

[97] Min K, Smuder AJ, Kwon OS et al. Mitochondrial-targeted antioxidants protect skeletal muscle against immobilization-induced muscle atrophy. J Appl Physiol (1985) 2011; 111: 1459-1466. doi:10.1152/ japplphysiol.00591.2011

[98] Powers SK, Hudson MB, Nelson WB et al. Mitochondria-targeted antioxidants protect against mechanical ventilation-induced diaphragm weakness. Crit Care Med 2011; 39: 1749-1759. doi:10.1097/CCM.0b013e3182190b62

[99] Hyatt H, Deminice R, Yoshihara T et al. Mitochondrial dysfunction induces muscle atrophy during prolonged inactivity: A review of the causes and effects. Arch Biochem Biophys 2019; 662: 49-60. doi:10.1016/j.abb.2018.11.005

[100] Jaber S, Petrof B], Jung B et al. Rapidly progressive diaphragmatic weakness and injury during mechanical ventilation in humans. Am J Respir Crit Care Med 2011; 183: 364-371. doi:10.1164/rccm.201004-0670OC

[101] Riley DA, Slocum GR, Bain JL et al. Rat hindlimb unloading: soleus histochemistry, ultrastructure, and electromyography. J Appl Physiol (1985) 1990; 69: 58-66. doi:10.1152/jappl.1990.69.1.58
[102] Busch WA, Stromer MH, Goll DE et al. Ca 2+ -specific removal of Z lines from rabbit skeletal muscle. J Cell Biol 1972; 52: 367-381. doi:10.1083/jcb.52.2.367

[103] Xiao YY, Wang MC, Purintrapiban J et al. Roles of mu-calpain in cultured L8 muscle cells: application of a skeletal muscle-specific gene expression system. Comp Biochem Physiol C Toxicol Pharmacol 2003; 134: 439-450

[104] Dayton WR, Goll DE, Zeece MG et al. A Ca2 +-activated protease possibly involved in myofibrillar protein turnover. Purification from porcine muscle. Biochemistry 1976; 15: 2150-2158. doi:10.1021/ bi00655a019

[105] Solomon V, Goldberg AL. Importance of the ATP-ubiquitin-proteasome pathway in the degradation of soluble and myofibrillar proteins in rabbit muscle extracts. J Biol Chem 1996; 271: 26690-26697. doi:10.1074/jbc.271.43.26690

[106] Plant PJ, Bain JR, Correa JE et al. Absence of caspase-3 protects against denervation-induced skeletal muscle atrophy. J Appl Physiol (1985) 2009; 107: 224-234. doi:10.1152/japplphysiol.90932.2008

[107] Siu PM, Alway SE. Mitochondria-associated apoptotic signalling in denervated rat skeletal muscle. J Physiol 2005; 565: 309-323. doi:10.1113/jphysiol.2004.081083

[108] Du J, Wang X, Miereles C et al. Activation of caspase- 3 is an initial step triggering accelerated muscle proteolysis in catabolic conditions. J Clin Invest 2004; 113: 115-123. doi:10.1172/JCl18330

[109] Chen M, He H, Zhan S et al. Bid is cleaved by calpain to an active fragment in vitro and during myocardial ischemia/reperfusion. J Biol Chem 2001; 276: 30724-30728. doi:10.1074/jbc.M103701200

[110] Garcia-Perez C, Roy SS, Naghdi S et al. Bid-induced mitochondrial membrane permeabilization waves propagated by local reactive oxygen species (ROS) signaling. Proc Natl Acad Sci USA 2012; 109: 4497-4502. doi:10.1073/pnas.1118244109

[111] Polster BM, Basanez G, Etxebarria A et al. Calpain I induces cleavage and release of apoptosis-inducing factor from isolated mitochondria. J Biol Chem 2005; 280: 6447-6454. doi:10.1074/jbc.M41326920

[112] Joza N, Oudit GY, Brown D et al. Muscle-specific loss of apoptosisinducing factor leads to mitochondrial dysfunction, skeletal muscle atrophy, and dilated cardiomyopathy. Mol Cell Biol 2005; 25: 10261-10272. doi:10.1128/MCB.25.23.10261-10272.2005

[113] Guo BS, Cheung KK, Yeung SS et al. Electrical stimulation influences satellite cell proliferation and apoptosis in unloading-induced muscle atrophy in mice. PLoS One 2012; 7: e30348. doi:10.1371/journal. pone. 0030348

[114] Badugu R, Garcia M, Bondada V et al. N terminus of calpain 1 is a mitochondrial targeting sequence. J Biol Chem 2008; 283: 34093417. doi:10.1074/jbc.M706851200

[115] Ni R, Zheng D, Xiong S et al. Mitochondrial Calpain-1 disrupts ATP synthase and induces superoxide generation in type 1 diabetic hearts: A novel mechanism contributing to diabetic cardiomyopathy. Diabetes 2016; 65: 255-268. doi:10.2337/db15-0963

[116] Smith IJ, Dodd SL. Calpain activation causes a proteasome-dependent increase in protein degradation and inhibits the Akt signalling pathway in rat diaphragm muscle. Exp Physiol 2007; 92: 561-573. doi:10.1113/expphysiol.2006.035790

[117] Smith IJ, Lecker SH, Hasselgren PO. Calpain activity and muscle wasting in sepsis. Am J Physiol Endocrinol Metab 2008; 295: E762-E771. doi:10.1152/ajpendo.90226.2008

[118] Chen HH, Liu P, Auger P et al. Calpain-mediated tau fragmentation is altered in Alzheimer's disease progression. Sci Rep 2018; 8: 16725. doi:10.1038/s41598-018-35130-y

[119] Harriss D], MacSween A, Atkinson G. Ethical Standards in Sport and Exercise Science Research: 2020 Update. Int J Sports Med 2019; 40: 813-817. doi:10.1055/a-1015-3123 